\title{
Performance Evaluation of Image Fusion Algorithms for Underwater Images-A study based on PCA and DWT
}

\author{
Ansar MK, Vimal Krishnan VR \\ Department of Computer Science, School of Mathematical and Physical Science \\ Central University of Kerala, Kasargod-671 314, India \\ (ansarmk07, vimallnair)@gmail.com
}

\begin{abstract}
In this paper, a comparative study between two image fusion algorithm based on PCA and DWT is carried out in underwater image domain. Underwater image fusion is emerged as one of the main image fusion area, here two or more images will be fused by retaining the most desirable characteristics of each underwater images. The DWT technique is used to decompose the input image into four frequency sub bands and the lowlow sub band images will be considered in fusion processing. In PCA method significant eigen values will be considered in fusion process to retain the important characteristics of the input images. The results acquired from both experiments are tabulated and compared by considering the statistical measures such as Peak Signal to Noise Ratio (PSNR), Mean Square Error (MSE) and Entropy. Results shows that underwater image fusion based on DWT outperforms the PCA based method.
\end{abstract}

Index Terms - Image Fusion, Image Enhancement, PCA, DWT, MSE, PSNR.

\section{INTRODUCTION}

Underwater image processing is emerged as one of the main research area of image processing. Especially it is widely used in ocean exploration, defense, and fish detection [1]. However, the quality of the underwater images is reduced because of the absorption and scattering effects of the underwater environment [2]. Also it may contain distortion and degradation in the form of noise, blur etc. [3]. Researchers come up with different techniques for improving the quality of underwater images. Image fusion is one such technique. This paper explain the performance evaluation of two algorithm based on PCA and DWT.

The image fusion is a branch of data fusion and it is the process of combining two or more images to form a single image [4]. So the fused image gives much better information than the original images [5][15]. The Fusion process will reduce the volume of data by creating compatible images with perception capability of human operator by completing image processing tasks like: image segmentation, object detection or target recognition [5].
Image fusion is used in the areas like defence [6], surveillance [7], target tracking [8], Medical Imaging [9][10], Biometrics [11], Robot vision, Aerial imaging and Satellite imaging [12][13][14] etc.

The Fusion process can be classified into three levels. They are pixel level image fusion, feature level image fusion; decision level image fusion [16][17]. The decision level and feature level fusions are high-level fusions that require more complex algorithms and more intensive computation. The pixel level fusion is the lowest level fusion that fuses the images from different physical channels pixel by pixel to enhance the features not complete in either channel [18]. Therefore, it requires less processing time and is found suitable for time critical image fusion applications such as underwater image processing specially for defence purpose.

The paper has been divided into five sections. Section II describes the principal component analysis. Discrete wavelet transform based fusion is discussed in Section III followed by experiment and comparative study in section IV. Conclusions are summarized in section V.

\section{PRINCIPAL COMPONENT ANALYSIS}

PCA is probably the most widespread multivariate statistical technique. Karl Pearson introduces it in 1901. Principal Component Analysis (PCA) is often used to reduce multidimensional data sets to lower dimensions for analysis. It reveals the internal structure of data in an unbiased way [19]. The PCA image fusion method uses the pixel values of all source images at each pixel location. Then adds a weight factor to each pixel value (it is known as standardized PCA). The average of the weighted pixel values will be used to produce fused image [20]. The optimal weighted factors are determined by the PCA technique.

PCA is very useful for understanding the variability in underwater image data set. Sometimes especially in defense application underwater images may contain large amount of information. It can be reduced by PCA without losing the information by compression. And also the PCA technique is useful for image encoding, image data compression and image enhancement [20]. 
PCA is implemented by using following mathematical procedure.

Step1: Select two underwater images with same resolution.

Step2: Adjust the image matrix by subtracting the mean from both original image matrixes. The mean can be found using the formula

$$
{ }_{i=1}^{n} \frac{x i}{n}
$$

Step3: Calculate the covariance of the image matrix. It can be found by using the formula.

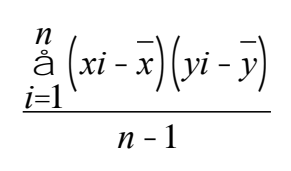

Step4: Calculate the eigenvectors and eigenvalues from the covariance matrix. The eigenvector will give most important data's.

Step5: Form the feature vectors by ordering eigenvalues based on their significance.

$$
\text { features vector }=\left(\operatorname{eig}_{1}, \text { eig }_{2}, \ldots \ldots \ldots, \text { eig }_{\mathrm{n}}\right)
$$

Step6: The fused image is formed by taking the transpose of the feature vector and multiplies it on the left of the original data set, transposed. That is

$$
\text { ImageMatrix }=(\text { RowFeatureVector } * \text { RowDataAdjust })
$$

\section{DESCRETE WAVELET TRANSFORM BASED FUSION}

Wavelet is a famous technique used for analyzing signals. It has the ability to preserve the time and frequency details of the images to be fused [22][23]. It provides a variety of channels representing the image feature by different frequency sub-bands. Li et al [22] and Chipman et al [24] introduced DWT into image fusion. The discrete 2-dimensional wavelet transform is computed by the recursive application of low pass and high pass filters in each direction of the input image followed by sub sampling [23][25]. The discrete wavelets transform (DWT) allows the image decomposition in different kinds of coefficients preserving the image information. When decomposition is performed, the approximation and detail component can be separated [15][16][26].

The DWT merges the coefficient to get the best result in the fused image. We can do it by considering the average of coefficient [19]. The average method and it is one of the basic methods to implement discrete wavelet fusion.

Here, two underwater images with same spatial resolution are used. The decomposition is achieved by applying DWT on both images. Only the coefficients at the same level and representation are fused. Final fused image is obtained by taking IDWT (Inverse Discrete Wavelet transform).

The procedure given below shows different steps to perform DWT on underwater images.

Step1: Select two underwater images with same resolution.

Step2: Apply decomposition using DWT on both input images.

Step3: Fuse each wavelet coefficient using average method.

Step4: perform IDWT to get the fused image.

\section{EXPERIMENT AND RESULT}

In order to measure the performance of the PCA and DWT fusion techniques, two underwater images with same resolution are used. The original images are in jpg format. The images of the scene 1 and scene 2 are given in fig. 1 and 2.

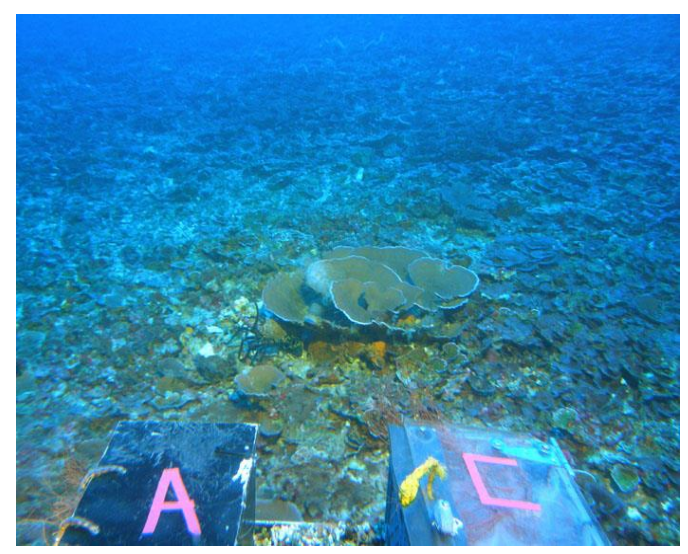

Fig 1. Underwater Image scene 1

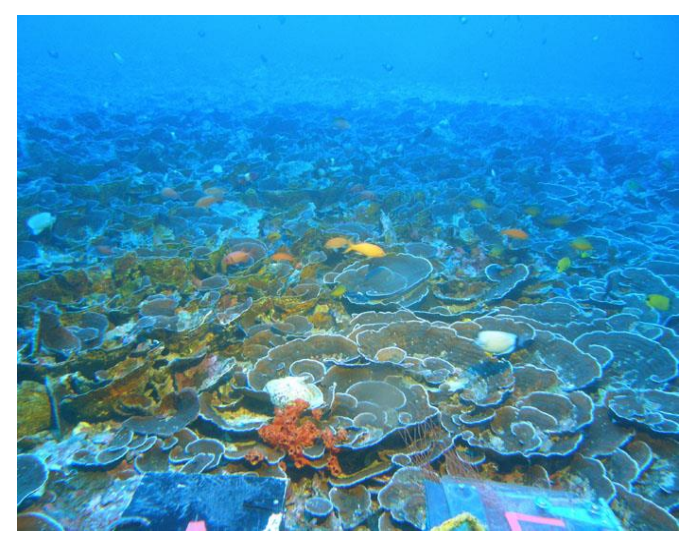

Fig 2. Underwater Image scene 2

The performance measuring properties such as entropy, mean square error and peak signal to noise ratio shows the improvement in the fused image for both methods. These are the commonly used statistical measures in assessing image fusion techniques. Mean Square Error and Peak Signal to Noise Ratio consider image as a special type of signal. Table 1 and 2 shows the measured values for both methods. 


\section{A. Entropy}

Entropy is a statistical measure of randomness. It can be used to characterize the texture of the input image. Entropy is defined as

$$
\operatorname{sum}(p \log 2(p))
$$

Where $\mathrm{p}$ contains the histogram counts returned from histogram of the image.

\section{B. MSE (Mean Square Error)}

The mean square error of an image can be finding out by using the following formulae.

$$
M S E=\frac{1}{m n}{ }_{i=0}^{m{ }^{1} n_{j} 1}[I(i, j) \quad K(i, j)]^{2}
$$

\section{PSNR (Peak Signal to Noise Ratio)}

The equation given below is used to find the PSNR between input image and fused image.

$$
P S N R=20 \log _{10}\left(M A X_{I}\right)-10 \log _{10}(M S E)
$$

These measures give only the global idea of the images. Also when assessing the performance of image fusion techniques using above measurements, we require the knowledge of both original image and fused image.

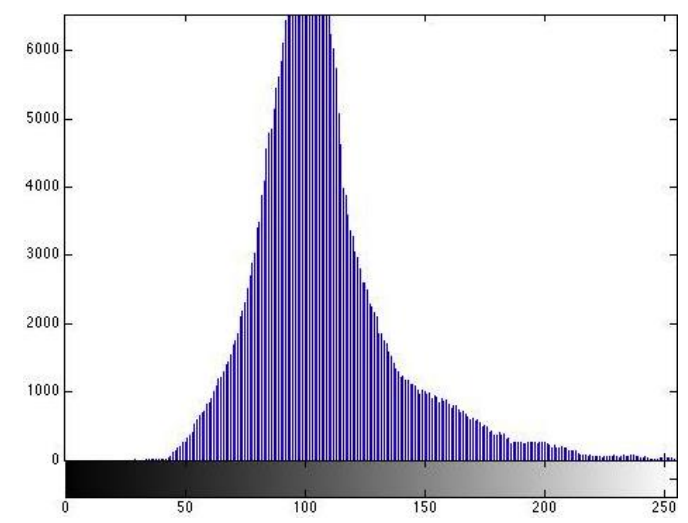

Fig 3. Histogram of Underwater image 1

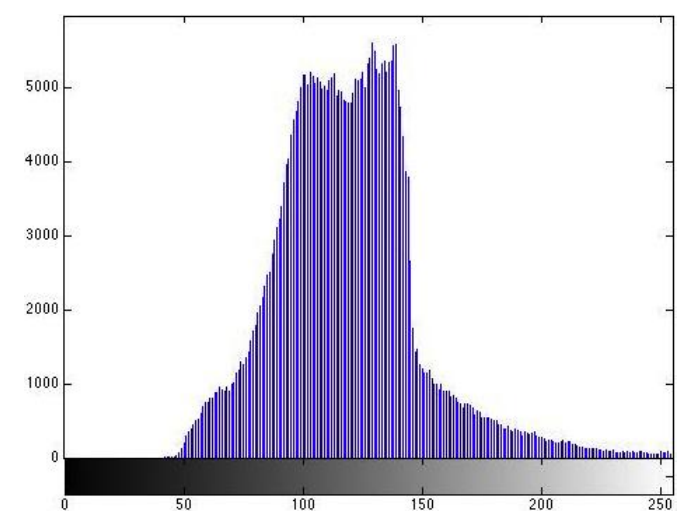

Fig 4. Histogram of Underwater image 2
Fig 3 and 4 above shows the histogram of the underwater images of scene 1 and 2 . The fused images of two scenes are given below.

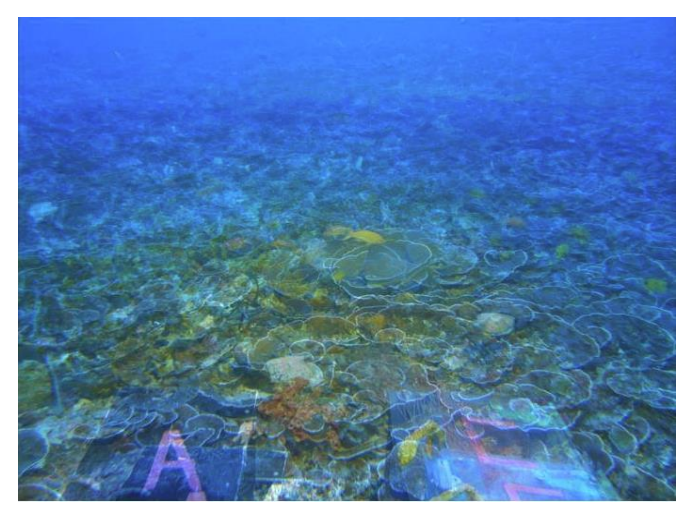

Fig 5. PCA fused image

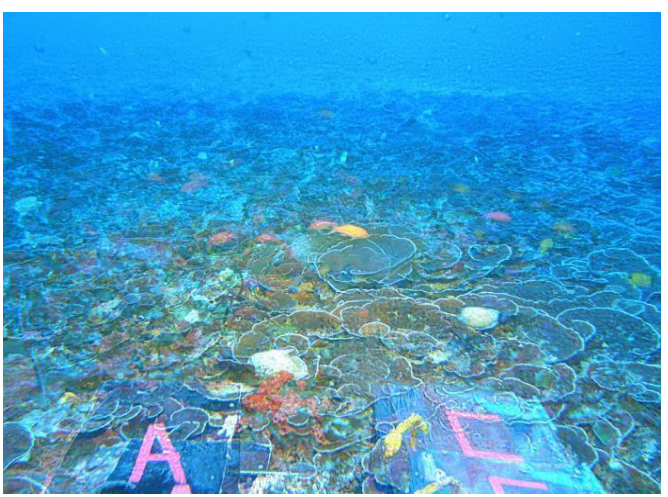

Fig 6. Wavelet fused image

PCA is a standard fusion technique based on the spatial domain, so it has got lower processing speed because of the presence of large amount of pixel level information. Where as in the case of wavelet, fusion takes place in the transform domain by combining the wavelet coefficients. That speedup the process and also produce better fused image. The histogram of the both methods is given in the fig. 7 and 8 .

In the case of under water images wavelet based approach is very useful, because we can fuse the images with different resolution. But it is not possible in standard PCA. Decomposition and fusing of coefficient helps to collect the information appropriately in DWT. Higher

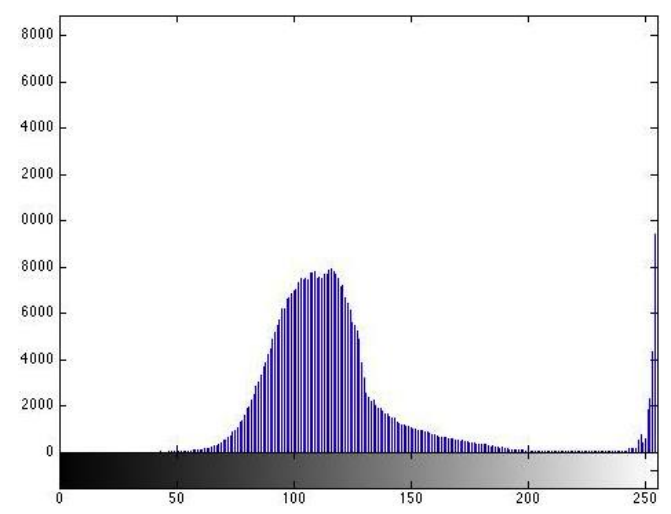

Fig 7. Histogram of PCA fused image 


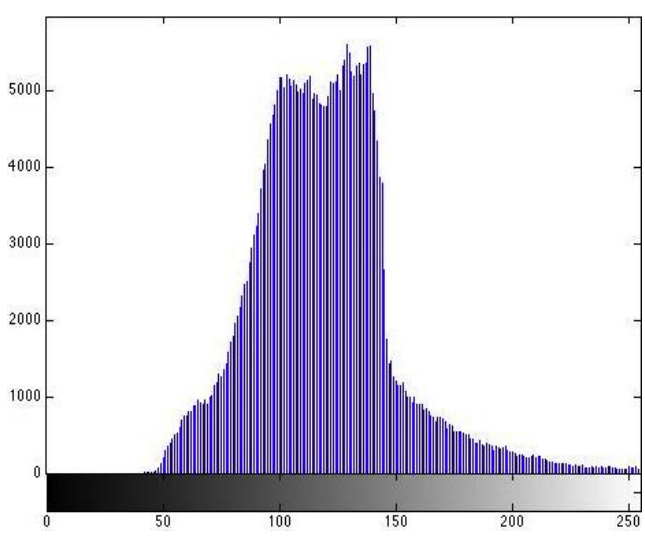

Fig 8. Histogram of wavelet-fused image

value of MSE value in PCA based fusion indicates the perseverance of spatial information. But it causes spectral degradation. That inversely affects the quality of the fused image. DWT out perform this problem by minimizing the spectral distortion. DWT produce higher PSNR value for fused image than PCA based fusion. It shows the higher quality of fused image.

We have got a maximum of 17.8574 for PSNR while comparing figure 2 and fused image in DWT based fusion. Where as in the case of figure 1 and fused image it is 16.3618 only. But in the case of PCA based fusion it is about 9.2990 and 9.5698 for figure 1 and figure 2 while comparing with fused image.

Table 1. Performance measures of PCA

\begin{tabular}{|l|l|l|l|l|l|}
\hline \multicolumn{2}{|c|}{ Entropy } & \multicolumn{2}{c|}{ MSE } & \multicolumn{2}{c|}{ PSNR } \\
\hline Fig 1 & 6.6799 & $\begin{array}{l}\text { Fig 1 } \\
\text { and } \\
\text { Fig 2 }\end{array}$ & $2.5603 \mathrm{e}+03$ & $\begin{array}{l}\text { Fig 1 } \\
\text { and } \\
\text { Fig 2 }\end{array}$ & 14.0479 \\
\hline Fig 2 & 6.8322 & $\begin{array}{l}\text { Fig1 } \\
\text { and } \\
\text { fused } \\
\text { image }\end{array}$ & $7.6416 \mathrm{e}+03$ & $\begin{array}{l}\text { Fig1 } \\
\text { and } \\
\text { fused } \\
\text { image }\end{array}$ & 9.2990 \\
\hline $\begin{array}{l}\text { Fusio } \\
\text { n } \\
\text { result }\end{array}$ & 5.7868 & $\begin{array}{l}\text { Fig2 } \\
\text { and } \\
\text { fused } \\
\text { image }\end{array}$ & $7.1796 \mathrm{e}+03$ & $\begin{array}{l}\text { Fig2 } \\
\text { and } \\
\text { fused } \\
\text { image }\end{array}$ & 9.5698 \\
\hline
\end{tabular}

Table 2. Performance measures of DWT

\begin{tabular}{|l|l|l|l|l|l|}
\hline \multicolumn{2}{|c|}{ Entropy } & \multicolumn{2}{c|}{ MSE } & \multicolumn{2}{c|}{ PSNR } \\
\hline Fig 1 & 6.6799 & $\begin{array}{l}\text { Fig 1 } \\
\text { and } \\
\text { Fig 2 }\end{array}$ & $2.5603 \mathrm{e}+03$ & $\begin{array}{l}\text { Fig 1 } \\
\text { and } \\
\text { Fig 2 }\end{array}$ & 14.0479 \\
\hline Fig 2 & 6.8322 & $\begin{array}{l}\text { Fig 1 } \\
\text { and } \\
\text { fused } \\
\text { image }\end{array}$ & $1.5028 \mathrm{e}+03$ & $\begin{array}{l}\text { Fig 1 } \\
\text { and } \\
\text { fused } \\
\text { image }\end{array}$ & 16.3618 \\
\hline $\begin{array}{l}\text { Fusio } \\
\mathrm{n} \\
\text { result }\end{array}$ & 6.8032 & $\begin{array}{l}\text { Fig 2 } \\
\text { and } \\
\text { fused } \\
\text { image }\end{array}$ & $1.0650 \mathrm{e}+03$ & $\begin{array}{l}\text { Fig 2 } \\
\text { and } \\
\text { fused } \\
\text { image }\end{array}$ & 17.8574 \\
\hline
\end{tabular}

\section{CONCLUSION}

In this paper performance of the two fusion methods such as PCA and DWT were compared statistically in underwater domain. Image fusion is performed to create a single enhanced image more suitable for different application. PCA primarily works with spatial domain and it is very useful for image fusion, data classification and dimensionality reduction. It has been found that wavelet based fusion techniques outperform the PCA fusion in spatial and spectral quality, especially in minimizing color distortion. Higher value of PSNR clearly shows it. So Wavelet based fusion with higher level of decomposition showed better performance in underwater images. In order to get better spatial and spectral resolution it is recommended to use both PCA and Wavelet together.

\section{ACKNOWLEDGEMENT}

The authors wish to thank Dr. Thasleema TM, Dr. Manjunath S, Dr. Abbas T.P, Mr. Kumar V, Mr. Sujith B, Ms. Daisy and Mr. Rajesh Kumar Assistant professors, Department of Computer Science, Central University of Kerala, India.

\section{REFERENCES}

[1] Raimondo Schettini; Silvia Corchs, "Underwater Image Processing: State of the Art of Restoration and Image Enhancement Methods". EURASIP Journal on Advances in Signal Processing 2010, 2010:746052.

[2] Shuai Fang; Rong Deng; Yang Cao, "Effective Single Underwater Image Enhancement by Fusion". Journal of computers, Vol. 8, No. 4, April 2013.

[3] Ancuti; Haber; Bekaert, "Enhancing underwater images and videos by fusion", Computer Vision and Pattern Recognition -21 June 2012.

[4] A. Ardeshir Goshtasby; Stavri Nikolov,"Image fusion: Advances in the state of the art," Volume 8, Issue 2, Pages 113-222 (April 2007).

[5] Petrică Turtoi; Niculae Guzulescu; Daniel Ţurcanu, "Image Fusion Based on Wavelet Transform", International Conference of Scientific Paper, AFASES 2013 Brasov, 23-25 May 2013.

[6] Toet A; Aguilare M; Waxman Am, "Fusion of visible and thermal imagery improves situation awareness", Volume 18, Issue 2, 30 December 1997, Pages 85-95.

[7] Snidaro, L; Luca Foresti, G; Niu, R; Varshney P.K, "Sensor fusion for video surveillance", Proceedings of the 7th International Conference on Information Fusion, Stockholm, Sweden, 2004, pp. 739-746.

[8] Sworder D, D; Boyd J.E; Clapp G.A, "Image fusion for tracking manoeuvring targets," International Journal of Systems Science, Vol.28, Issue 1, 1997, pages 1-14.

[9] Hill, D; Edwards,P; Hawkes, D; "Fusing medical images," Image Processing , Vol.6, Issue 2, 1994, pages 109-113.

[10] CYN Dwith; Vivek Angoth; Amarjot Singh, "Wavelet Based Image Fusion for Detection of Brain Tumor", IJIGSP, vol.5, no.1, pp.25-31, 2013.DOI: 10.5815/ijigsp.2013.01.04.

[11] Anouar Ben Khalifa; Najoua Essoukri BenAmara, "Contribution to the Fusion of Biometric Modalities by the Choquet Integral", IJIGSP, vol.4, no.10, pp.1-7, 2012.

[12] Goshtasby A; "2-D and 3-D Image Registration for Medical, Remote Sensing, and Industrial Applications," Wiley Press, 2005.

[13] Pohl,C; Genderen J,L, "Multisensor image fusion in remote sensing: concepts, methods, and applications," International Journal of Remote Sensing, Vol.19, Issue 5,1998, pages 823-854. 
[14] Simone G; Farina A; Morabito F,C; Serpico S,B; Bruzzone L, "Image fusion techniques for remote sensing applications," Information Fusion, Vol. 3,2002, pages 315.

[15] Varsha patil; Deepali sale; Joshi M.A, "Image Fusion Methods and Quality Assessment Parameters".

[16] Bedi S.S; Khandelwal Rati, "Comprehensive and Comparative Study of Image Fusion Techniques", International Journal of Soft Computing and Engineering (IJSCE) ISSN: 2231-2307, Vol.3, Issue-1, March 2013.

[17] Mrinal Kanti Bhowmik, Kankan Saha, Sharmistha Majumder, "Thermal Infrared Face Recognition-a Biometric Identification Technique for Robust Security System".

[18] Tumpa Dey, “A Survey on Different Fusion Techniques of Visual and Thermal Images for Human Face Recognition", International Journal of Electronics Communication and Computer Engineering Vol. 4, Issue 6, NCRTCST -2013, ISSN 2249-071X.

[19] Zhijun Wang, Djemel Ziou, Costas Armenakis, Deren Li, and Qingquan Li in "A Comparative Analysis of Image Fusion Methods". IEEE transactions on geoscience and remote sensing, vol.43, no.6, June 2005.

[20] Lanir J, "Comparing multispectral image fusion methods for a target detection task," Optical Engineering, vol. 46, pp. 66402-1, 2007.

[21] Nirosha Joshitha J; Medona Selin R,"Image Fusion using PCA in Multifeature Based Palmprint Recognition," International Journal of Soft Computing and Engineering (IJSCE) ISSN: 2231-2307, Vol.2, Issue-2, and May 2012.

[22] Hui Li; Manjunath, B.S.; Mitra, S.K, "Multi-sensor image fusion using the wavelet transform," Image Processing, 1994. Proceedings. ICIP-94., IEEE International Conference, vol.1, pp.51, 55 vol.1, 13-16 Nov 1994.

[23] Gonzalo Pajares; Jesus Manuel de la cruz, "A wavelet based image fusion tutorial," Pattern Recognition, Vol. 37, 2004, pages 1855-1872.

[24] Chipman L.J; Orr Y.M; Graham L.N, "Wavelets and image fusion," Proceedings of the International
Conference on Image Processing, Washington, USA, 1995, pp. 248-251.

[25] Shefer r; Malhi M; Shenhar A, "Wave distortion correction using cross correlation," Technion, Israel Institute of Technology.

[26] Amarjot Kaur; Sunil Khullar "Image Fusion using HIS, PCA and Wavelet Technique," International Journal of Computer Science and Communication Engineering Vol. 2 Issue 2 (May 2013 Issue).

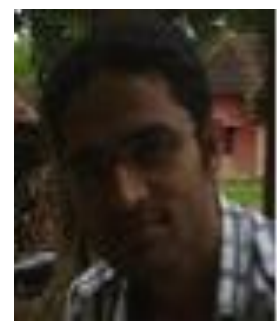

Ansar MK is a Master of Science student with Specialization in Computational Intelligence at Central University of Kerala, India. $\mathrm{He}$ completed Bachelors in Computer Science from Kannur University Kerala. $\mathrm{He}$ is the founder and chairman of 'innovDREAMZ', a potential research group of students who works together to promote science research. His research interests include Image fusion, Computer Vision, Pattern Recognition, Data Mining etc.

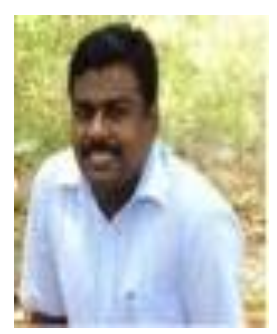

Vimal Krishnan VR is Assistant professor at Central University of Kerala, India. He completed his Masters in Software Science from Periyar University and doing $\mathrm{PhD}$ in Speech Processing at Kannur University. $\mathrm{He}$ is a Free Software and Knowledge Freedom Activist. His research interests include Speech Recognition, Artificial Neural Network, Wavelet, Signal Processing, and Emotion Recognition etc.

How to cite this paper: Ansar MK, Vimal Krishnan VR,"Performance Evaluation of Image Fusion Algorithms for Underwater Images-A study based on PCA and DWT", IJIGSP, vol.6, no.12, pp. 65-69, 2014.DOI: 10.5815/ijigsp.2014.12.09 